\title{
Molecular hybridization of polydimethylsiloxane with zirconia for highly gas permeable membranes
}

\author{
Roman Selyanchyn $^{1^{*}}$ and Shigenori Fujikawa $a^{1,2,3^{*}}$ \\ ${ }^{1}$ WPI International Institute for Carbon-Neutral Energy Research (WPI-I²CNER), Kyushu University, 744 \\ Motooka, Nishi-ku, Fukuoka 819-0395, Japan \\ ${ }^{2}$ Center for Molecular Systems (CMS), Kyushu University, Kyushu University, 744 Motooka, Nishi-ku, \\ Fukuoka 819-0395, Japan \\ ${ }^{3}$ NanoMembrane Technologies Inc., 4-1, Kyudai-Shimachi, Nishi-ku, Fukuoka, 819-0388, Japan
}

\section{Corresponding Authors}

* Tel/Fax: +81 92 8026717. E-mail: romanselyanchyn@i2cner.kyushu-u.ac.jp

* Tel/Fax: +8192 8026872. E-mail: fujikawa.shigenori.137@m.kyushu-u.ac.jp

\section{Membrane fabrication and assembly}

Membranes were fabricated according to the schematic procedure given in Fig. S1. Simple soda-lime glass $(5 \times$ $5 \mathrm{~cm}$ ) were used as substrates for the membranes fabrication. Substrate is first cleaned by washing in water and acetone. Then, treatment by oxygen plasma $(55 \mathrm{~W}, 10 \mathrm{cc} / \mathrm{min}, 10 \mathrm{~Pa})$ for 3 minutes was used to complete the cleaning and activate the surface. $15 \mathrm{wt} \%$ aqueous solution of PSS is spin-coated (3000 rpm for 40 seconds) on the activated glass to form ca. $500 \mathrm{~nm}$ PSS sacrificial layer. After spin-coating substrate with PSS layer is dried on the hot plate $\left(5 \mathrm{~min}\right.$ at $120^{\circ} \mathrm{C}$ ). Subsequently, border was formed on the PSS/glass using $3 \mathrm{~mm}$ thick commercially aquired silicone sheet glued by the aid of Sylgard 184 PDMS. To form a hybrid membrane the precursor solution was casted on the PSS/glass, covered with glass petri dish followed by heat treatment. After heating is completed membrane could bedetached from the glass after PSS dissolution in water. This procedure allows to avoid the mechanically rough step of membrane peeling from the glass and therefore allows fabrication of the membranes with higher quality.
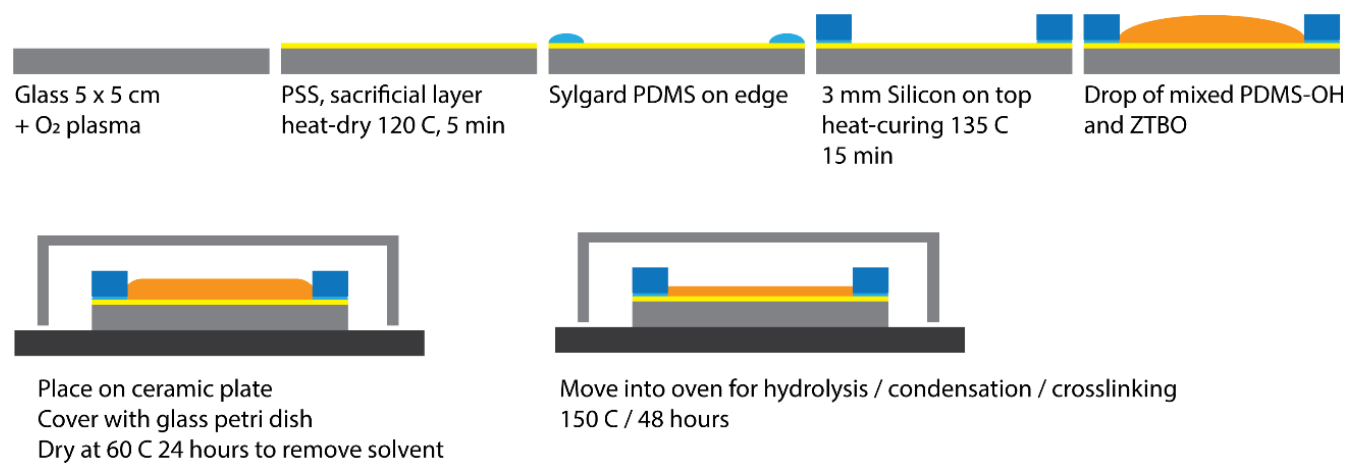

Move into oven for hydrolysis / condensation / crosslinking $150 \mathrm{C} / 48$ hours

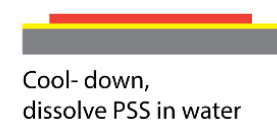

\section{ZrO2@PDMS}

casted membrane

Figure S1. Schematic procedure of the hybrid $\mathrm{ZrO}_{2} @ \mathrm{PDMS}$ membrane fabrication by casting on sacrificial PSS layer with subsequent detachment in water (upon the dissolution of PSS). 
For the gas permeation tests, $20 \mathrm{~mm}$ circle were cut using the hydraulic punch. Membranes were assembled for gas permeability testing according to the procedure shown in Fig. S2. Two layers of adhesive aluminum tape were used to ensure that there are no leaks possible around the membrane. Active area was set to $0.785 \mathrm{~cm}^{2}$.
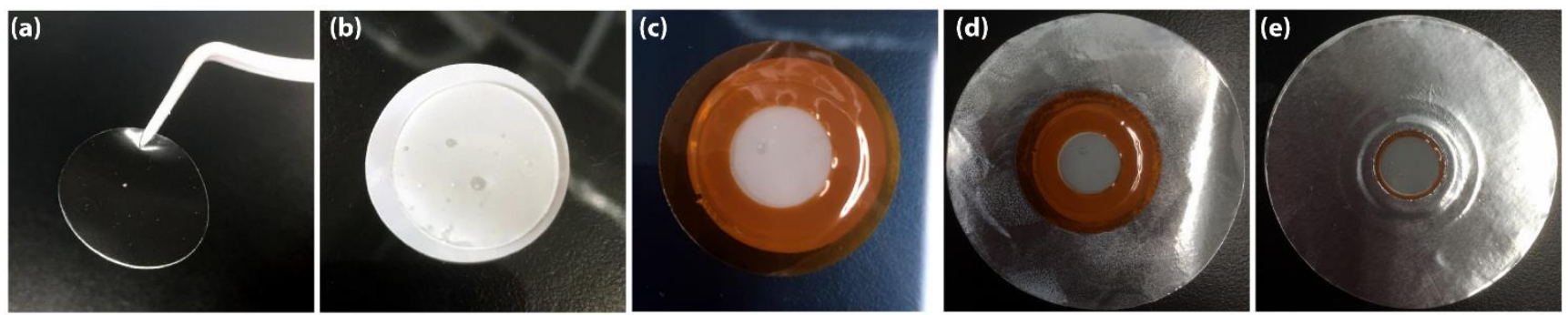

Figure S2. Membrane assembly for the gas permeability measurement: (a) thick casted membrane $d=2 \mathrm{~cm}$; (b) placed on the porous support (etched polycarbonate $1.2 \mu \mathrm{m}$ surface pores); (c) membrane area masked using a Kapton ${ }^{\circledR}$ tape with $1 \mathrm{~cm}$ opening; (d) membrane placed centered on the $100 \mu \mathrm{m}$ thick aluminum tape with $1 \mathrm{~cm}$ opening; (e) membrane covered with one more aluminum tape on the top surface with the $1.3 \mathrm{~cm}$ internal opening. The final membrane is sandwiched between two aluminum layers ensuring there is no leakage of the gas other than pressure difference driven permeation through the membrane.

\section{Experimental setup for gas transport measurement}

The part of the machine where test gas is sampled (GTR-31AKU) uses differential-pressure method for film permeability testing where gas permeation is induced by the vacuuming of the permeate side and extra pressure applied at the feed side, Fig. S3. Total applied pressure difference was equal to $0.2 \mathrm{MPa}$. In order to obtain gas concentration sufficient for the reliable detection by gas chromatography the sample collection time after vacuuming the permeate side of the membrane was set to 1 minute. The gas collected in the permeation cell is automatically transferred to a gas chromatograph where the volume of permeated gas is detected by comparison with the calibration sample. A gas chromatograph (Yanaco G3700T, Japan) is equipped with a thermal conductivity detector (TCD), and the appropriate carrier gas was used for different gases permeability measurement (He-carrier for $\mathrm{CO}_{2}, \mathrm{~N}_{2}, \mathrm{O}_{2}$ or Ar-carrier for $\mathrm{H}_{2}$ detection).

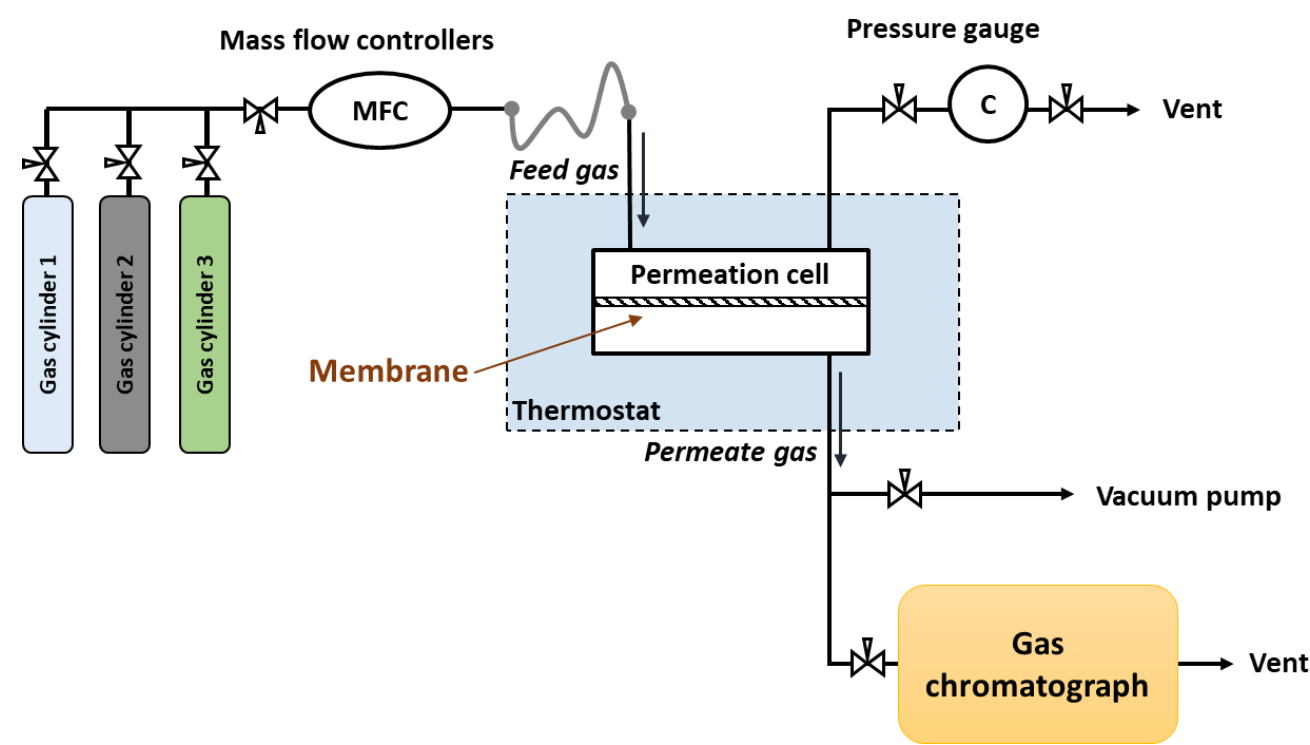

Figure S3. Schematic diagram of apparatus for measurement of gas flow through the membrane gas chromatograph (GTR-TEC equipment) for both single and mixed dry gas permeation tests. 


\section{Characterization}

\subsection{Elemental composition by XPS}
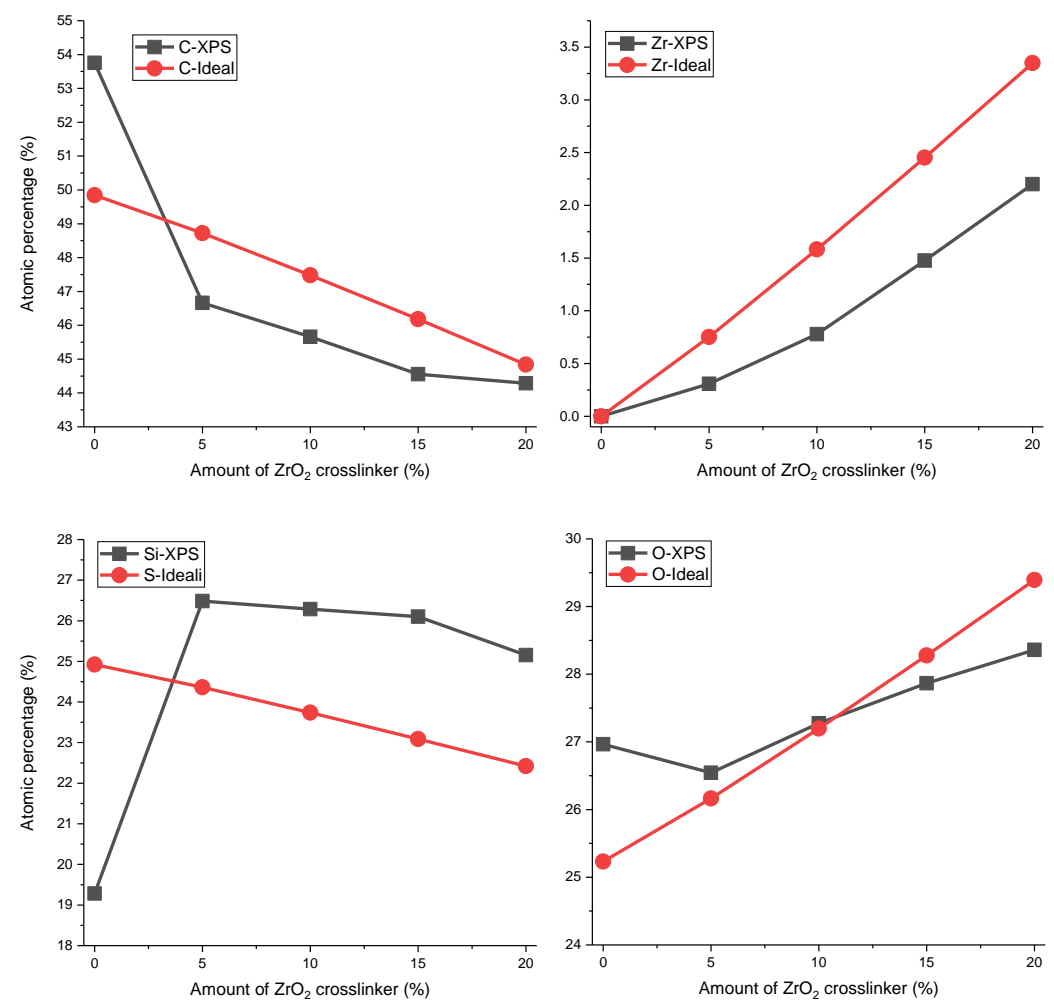

Figure S4. Comparison of the XPS derived elemental composition (black line) with the ideal elemental composition assuming complete hydrolysis of $\mathrm{ZrTBO}$ to $\mathrm{ZrO}_{2}$.

\subsection{Mechanical properties of the membranes assessed by nanoindentation}

The UNHT used in the study utilizes the integrated reference system, in which the sample is placed on the rigid sample holder to prevent a possible sinking of the reference system into the soft viscoelastic films [R1]. When the reference tip touches the surface, an indenter tip moves into the sample to be tested, applying an increasing normal load. The position of the indenter relative to the sample surface is monitored with a differential capacitive sensor. For nanoindentation tests, $\mathrm{ZrO}_{2} @ \mathrm{PDMS}$ nanocomposite films, as well as PDMS reference, were cast on a glass supports. In order to avoid the influence of the support on the measurement result, the films were of sufficient thickness (exceeding 20 $\mu \mathrm{m})$. Loading and unloading were done in a force controlled mode with a linear load increase. Maximum load used for all materials was set to $0.5 \mathrm{mN}$.

Typical nanoindentation curves of $\mathrm{ZrO}_{2} @ \mathrm{PDMS}$ composites with different $\mathrm{ZrO}_{2}$ content as well as a reference PDMS material are given in Fig.S5a. As seen, the indentation depth at the maximum load was significantly different for materials with different $\mathrm{ZrO}_{2}$ content, indicating the different mechanical properties. Compared to the reference PDMS film, 5 and $10 \% \mathrm{ZrO}_{2}$ loaded composites showed larger indentation depths while $15 \%$ and $20 \%$ demonstrated lower indentation depth at the same load. Such results indicate that $\mathrm{ZrO}_{2}$ does not play a role of simple physical filler in PDMS, because the incorporation of inorganic fillers usually promotes the hardness increase in polymer matrices scalable with the amount added [R1]. Mechanical properties that can be estimated from the nanoindentation tests are listed in Table S1 and also graphically depicted in the Figs. S5b. Here we can see how drastically amount of $\mathrm{ZrO}_{2}$ influences the properties that are changed exponentially with the increase of the metal oxide content. Compared to the reference PDMS, $10 \%$ and $15 \%$ hybrids are more close to it in terms of hardness and 
modulus. The extreme materials with $5 \% \mathrm{ZrO}_{2}$ and $20 \%$ are respectively much more soft and rigid compared to PDMS. The values of hardness and modulus obtained for PDMS were close to that already reported in the literature [R3]. Based on these results 10\%- $\mathrm{ZrO}_{2} @ \mathrm{PDMS}$ and 15\%- $\mathrm{ZrO}_{2} @ \mathrm{PDMS}$ nanocomposites are the most suitable material for membrane fabrication from the point of view of mechanical properties.

(a)

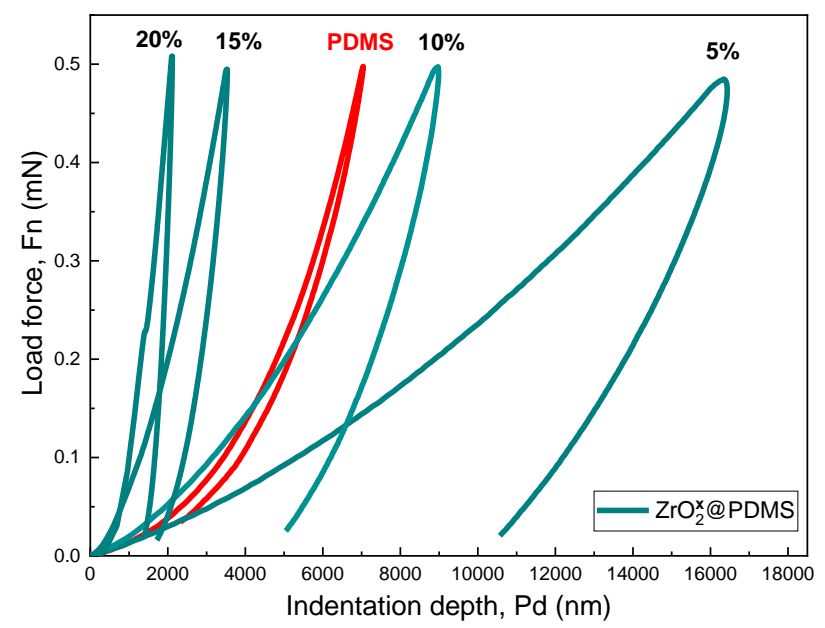

(b)

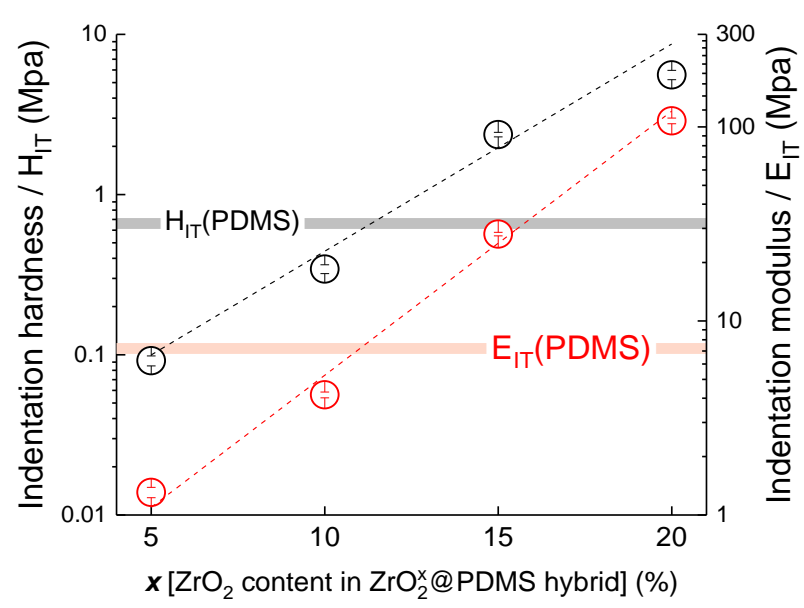

Figure S5. (a) Load vs. displacement curves measured in nanoindentation testing of the developed nanocomposites compared to the PDMS reference film, (b) indentation hardness and modulus correlation with $\mathrm{ZrO}_{2}$ content in the nanocomposite membranes and comparison with the Sylgard 184 PDMS.

Table S1. Mechanical properties of $\mathrm{ZrO}_{2} @$ PDMS nanocomposites measured by nanoindentation.

\begin{tabular}{cccc}
\hline $\begin{array}{c}\mathrm{ZrO}_{2} \text { loading, } \\
\mathbf{w t} \%\end{array}$ & $\begin{array}{c}\text { Indentation hardness (O\&P), } \\
{[\mathbf{M P a}]}\end{array}$ & $\begin{array}{c}\text { Indentation modulus (O\&P), } \\
{[\mathbf{M P a}]}\end{array}$ & $\begin{array}{c}\text { Plain strain modulus (O\&P), } \\
{[\mathbf{M P a}]}\end{array}$ \\
\hline $\mathbf{0}^{*}$ & $0.66 \pm 0.05$ & $5.41 \pm 0.20$ & $7.22 \pm 0.27$ \\
\hline $\mathbf{5}$ & $0.09 \pm 0.01$ & $1.31 \pm 0.08$ & $1.74 \pm 0.11$ \\
\hline $\mathbf{1 0}$ & $0.34 \pm 0.02$ & $4.16 \pm 0.14$ & $5.55 \pm 0.19$ \\
\hline $\mathbf{1 5}$ & $2.37 \pm 0.08$ & $28.1 \pm 0.6$ & $37.4 \pm 0.8$ \\
\hline $\mathbf{2 0}$ & $5.59 \pm 0.38$ & $107.5 \pm 3.5$ & $143.3 \pm 5.8$
\end{tabular}

${ }^{*}$ Loading $0 \%$ stands for the reference standard PDMS film prepared form commercial Sylgard 184 kit 
3.3. Small-angle X-ray scattering

(a)

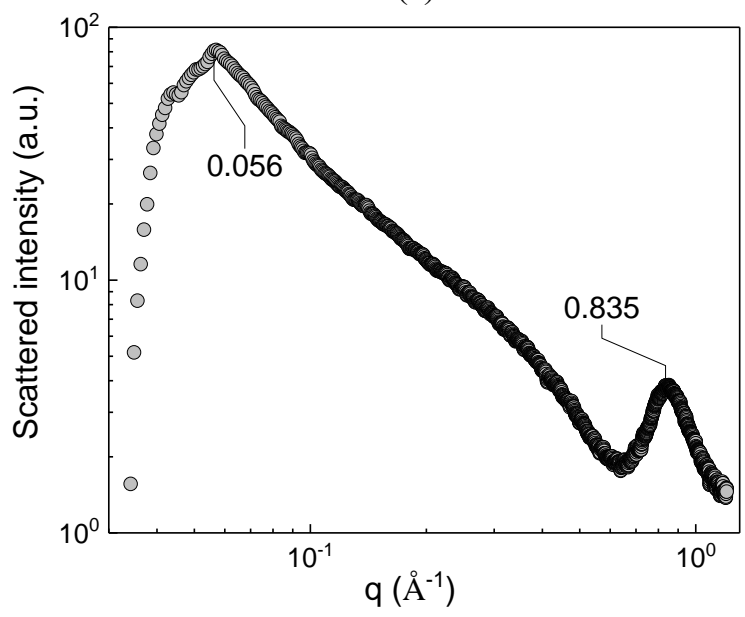

(b)

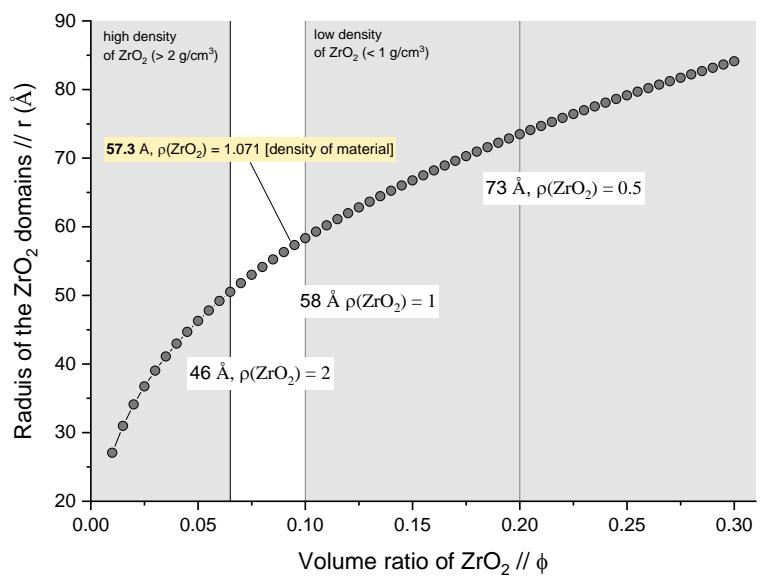

Fig. S6. (a) SAXS profile $\left(\lambda=1.54 \AA\right.$ ) of the casted 10\%- $\mathrm{ZrO}_{2} @$ PDMS membrane with indicated $\mathrm{q}_{\max }$ peaks $(0.835$ and $0.056 \AA^{-1}$ ) originating from PDMS and $\mathrm{ZrO}_{2}$ nanodomains respectively; (b) Estimation of the radius of $\mathrm{ZrO}_{2}$ domains depends on the density of $\mathrm{ZrO}_{2}$ in the nanocomposite. Densities in the grey area $\left(<1 \mathrm{~g} \cdot \mathrm{cm}^{-3}\right)$ are less realistic, as well as higher densities $\left(>2 \mathrm{~cm}^{-3}\right)$ that are characteristic for the crystalline forms of $\mathrm{ZrO}_{2}$. Crystalline form is also unlikely the case for our materials due to nature of fabrication (including only mild thermal treatment).

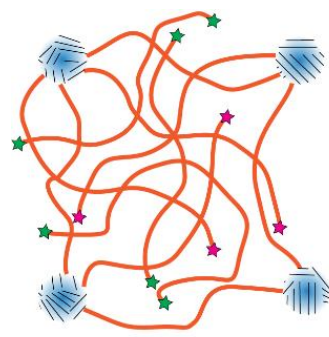

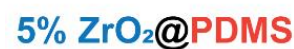

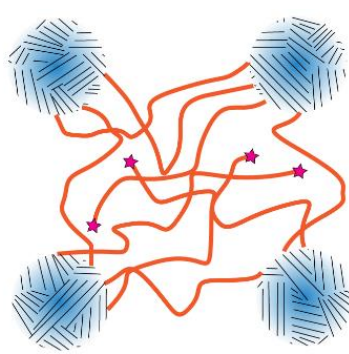

$10 \% \mathrm{ZrO}_{2} @ \mathrm{PDMS}$

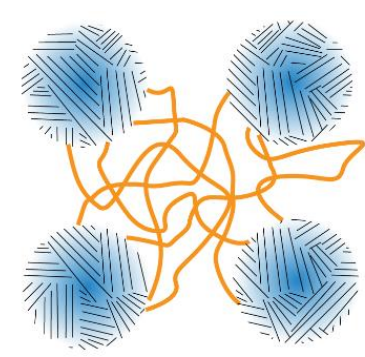

$15 \% \mathrm{ZrO}_{2} @ \mathrm{PDMS}$

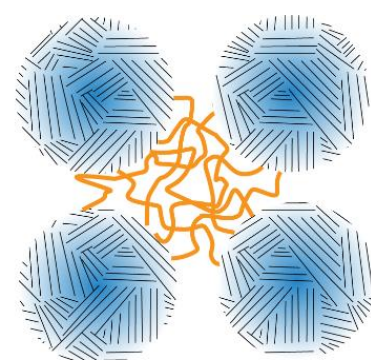

$20 \% \mathrm{ZrO}_{2} @ \mathrm{PDMS}$

Figure S7. Schematic structure of the developed nanocomposites based on the characterization and gas permeation data, depending on the amount of $\mathrm{ZrO}_{2}$ crosslinker.

\section{References}

[R1] Nohava, J.; Randall, N. X.; Conté, N. Novel Ultra Nanoindentation Method with Extremely Low Thermal Drift: Principle and Experimental Results. J. Mater. Res. 2009, 24 (3), 873-882. [R2] Ji, X.; Hampsey, J. E.; Hu, Q.; He, J.; Yang, Z.; Lu, Y. Mesoporous Silica-Reinforced Polymer Nanocomposites. Chem. Mater. 2003, 15 (19), 3656-3662.

[R3] Alisafaei, F.; Han, C. S. Indentation Depth Dependent Mechanical Behavior in Polymers. Adv. Condens. Matter Phys. 2015, Art.ID 391579, 1-20. 